\title{
Infant with MRSA necrotizing fasciitis
}

This article was published in the following Dove Press journal:

Research and Reports in Neonatology

2 May 2014

Number of times this article has been viewed

\author{
Maria Panglao Rajan' \\ Pinkal Patel' \\ Lori Cash' \\ Anjali Parish ${ }^{2}$ \\ Scott Darby' \\ Jack $\mathrm{Yu}^{3}$ \\ Jatinder Bhatia'
}

'Department of Pediatrics, Children's Hospital of Georgia, Augusta, GA,

USA; ${ }^{2}$ Medical Center of Central

Georgia, Augusta, GA, USA;

${ }^{3}$ Department of Plastic Surgery,

Children's Hospital of Georgia,

Augusta, GA, USA
Correspondence: Pinkal Patel

Children's Hospital of Georgia,

II 20 I5th Street, Augusta,

GA 309/2, USA

Email pipatel@gru.edu
Abstract: This is an unusual case of necrotizing fasciitis caused by methicillin resistant Staphylococcus aureus in this premature infant, which highlights severity, rapid progression of this disease and shows outcome if intervention is initiated at an early stage. This case also highlights one of the possible serious complications of percutaneous inserted central catheter (PICC) line, which can be life threatening.

Keywords: necrotizing fasciitis, methicillin resistant Staphylococcus aureus, PICC, premature infant

\section{Introduction}

This is a case of a 7-day-old premature infant with necrotizing fasciitis of the right upper extremity caused by methicillin resistant Staphylococcus aureus (MRSA) associated with a percutaneous inserted central catheter (PICC) line.

\section{Case presentation}

This baby was born at 30 weeks gestational age to a 28 -year-old Gravida 2 Para 1 mother with negative prenatal serologies, via vaginal delivery. The Apgar score was 7 and 8 , at 1 and 5 minutes, respectively. The mother had prolonged premature rupture of the membrane ( 92 hours) with clear fluid and received intrapartum ampicillin. The infant was intubated and received surfactant at birth, and was extubated to continuous positive airway pressure on which he remained for the first 6 days of life. A right arm PICC line was placed on the third day of life. On day of life 6, erythema and swelling were noted at the PICC line site. The right arm PICC line was removed and a new PICC line was placed in the left arm. The infant was noted to be febrile (temperature of $38.5^{\circ} \mathrm{C}$ ). A complete blood count, C reactive protein ([CRP] $4.2 \mathrm{mg} / \mathrm{dL}$ ), and a peripheral blood culture were obtained and the infant was started on intravenous vancomycin ( $15 \mathrm{mg} / \mathrm{kg} /$ dose every 12 hours) and gentamicin (4 mg/kg/day). On day of life 7, right hand and arm erythema and swelling increased which coincided with a positive blood culture for gram positive cocci, and as a result clindamycin $(5 \mathrm{mg} /$ $\mathrm{kg} /$ dose q12hr) was added. CRP increased to 23 . The infant developed respiratory distress and required reintubation. A Doppler study of the right hand and arm showed an abscess in the right mid-arm. A pediatric orthopedic surgeon was consulted and the abscess was incised and drained. A magnetic resonance image of the right upper extremity obtained from an external facility revealed extensive tissue swelling and mild diffuse enhancement indicative of necrotizing fasciitis. The infant was then transferred for further management. 


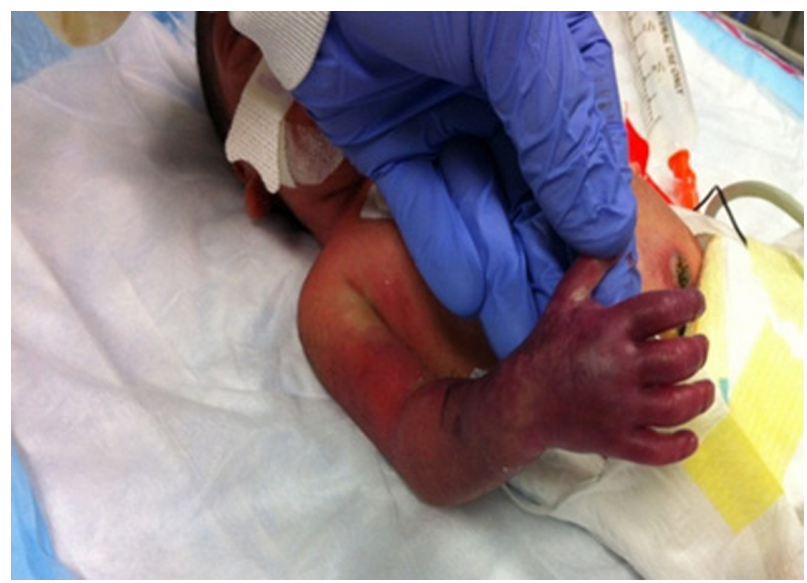

Figure I Marked erythema and swelling on the right hand, arm, and forearm. Note: There is erythema at the periumbilical region.

Physical examination on admission to our institution noted tense swelling proceeding from the right hand to the ipsilateral hemithorax with significant erythema and discoloration of the right forearm. Perfusion of the right hand was poor with prolonged capillary refill time and absent radial and ulnar pulses (Figure 1).

Laboratory evaluation revealed thrombocytopenia (platelet count of 83,000), bandemia (17\%), and elevated CRP (15). In addition, the infant had elevated prothrombin time, partial thromboplastin time, and D-dimers which are evident of disseminated intravascular coagulation. The diagnosis of compartment syndrome was made and the infant underwent immediate fasciotomy of the right forearm and arm. Extensive irrigation was done using standard irrigant. All wounds were further explored to ensure no undrained area and wounds were left completely open. He was noted to have nonviable subcutaneous tissue, a large amount of cloudy fluid, and pale muscles intraoperatively which were debrided. A wound culture was sent. Vancomycin, gentamicin, and clindamycin were continued and serial peripheral blood cultures obtained. The wound culture was positive for MRSA (sensitive for vancomycin, rifampin, bactrim, and linezolid, and resistant for clindamycin, erythromycin, and ciprofloxacin). MRSA was persistently isolated from the peripheral blood cultures for 3 days necessitating the addition of rifampin $(10 \mathrm{mg} / \mathrm{kg} /$ day q12hr). He required multiple blood products and dopamine infusion as supportive measures.

In total, the infant received rifampin for 9 days and vancomycin for 3 weeks following the first negative blood culture. He received daily dressing changes and wound care with limited return of function noted at 3 weeks after fasciotomy (Figure 2).

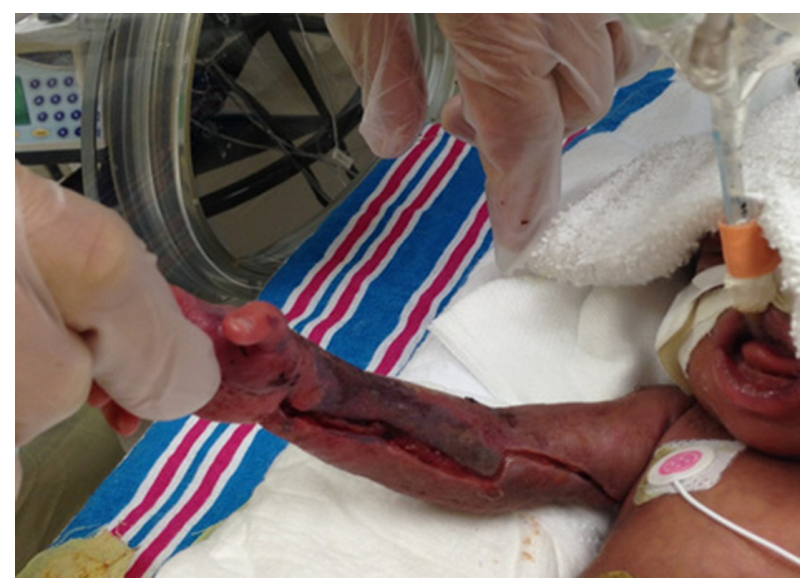

Figure 2 Healing fasciotomy wound.

Note: Note the pink colored thumb and fingers.

\section{Discussion}

We present this case to highlight the unusual causative organism in this age group, the rapidity with which the disease progressed, and the surgical intervention required. This infant had necrotizing fasciitis of the right hand and arm due to MRSA secondary to central line infection.

Necrotizing fasciitis is a rapidly progressing infection of skin and soft tissues which requires immediate administration of broad spectrum antibiotics and surgical debridement. It is generally caused by group A streptococcus, clostridium perfringens, or a mixture of anaerobic and aerobic pathogens. Staphylococcus is commonly isolated from mixed infections. Necrotizing fasciitis caused solely by MRSA in the newborn is extremely unusual and only a few cases are reported in the literature. ${ }^{1}$

Infection typically spreads along the muscle fascia due to its relatively poor blood supply. Initially, the overlying tissue can appear unaffected and can make necrotizing fasciitis difficult to diagnose without surgical intervention. Marked swelling and edema may produce compartment syndrome especially involving extremities with complicating myonecrosis requiring urgent fasciotomy. ${ }^{1}$ Most neonates with necrotizing fasciitis are systemically ill with fever, tachycardia, hypotension, and irritability. Diagnosis is usually based on signs and symptoms; laboratory findings may aid or support the diagnosis. Abnormalities may include leukocytosis with a marked left shift and elevated CRP. These laboratory findings, the clinical finding described above, and high index of suspicion should prompt immediate surgical exploration without delaying for radiological studies. Blood cultures and tissue specimens from surgical exploration should be sent for culture. Broad spectrum antibiotics (eg, vancomycin, gentamicin, or clindamycin) and supportive care are the mainstay 
of treatment along with surgical debridement. ${ }^{2}$ Empiric antibiotics should cover aerobic and anaerobic organisms and definite antimicrobial treatment should be targeted based on culture and the sensitivity of the organism. A good nutrition status should be maintained throughout the illness. Mortality rates can reach up to $60 \%$ even with treatment. ${ }^{1}$

\section{Disclosure}

The authors report no conflict of interest in this work.

\section{References}

1. Nazir Z. Necrotizing fasciitis in neonates. Pediatr Surg Int. 2005;21(8): 641-644.

2. Edlich RF, Cross CL, Dahlstrom JJ, Long WB 3rd. Modern concepts of the diagnosis and treatment of necrotizing fasciitis. J Emerg Med. 2010;39(2):261-265.

\section{Publish your work in this journal}

Research and Reports in Neonatology is an international, peer-reviewed, open access journal publishing original research, reports, editorials, reviews and commentaries on neonatal health. The manuscript management system is completely online and includes a very quick and fair peer-review system. Visit http://www.dovepress.com/testimonials.php to read real quotes from published authors. 